\title{
Dual-Frequency Broadband Antenna for Mobile Device Applications
}

\author{
Ting Zhang, Ronglin Li \\ South China University of Technology \\ Guangzhou 510641, China \\ zt20041050063@126.com
}

\author{
Manos M. Tentzeris \\ Georgia Institute of Technology \\ Atlanta, GA 30308, USA \\ etentze@ece.gatech.edu
}

\begin{abstract}
This paper proposes a low profile monopole antenna which achieves a dual-frequency broadband operation at 900 MHz with a bandwidth of $\sim 42 \%(790 \sim 1215)$ and at $1900 \mathrm{MHz}$ with a bandwidth $\sim 53 \% \quad(1710 \sim 3000 \mathrm{MHz})$. The proposed antenna has an inverted $F$ strip and a meandered strip directly fed by the microstrip line on the front side and has an $\mathrm{S}$ strip terminated at a ground plane. The lower sections of the inverted $F$ strip and the $S$ strip overlap and form a two-strip monopole. The mutual coupling of the two-strip lines has a significant effect on the bandwidth enhancement. The Antenna structure is described in detail and its performance is validated by experimental results.
\end{abstract}

Keywords- Broadband antenna, dual-frequency antenna, mobile communications.

\section{INTRODUCTION}

Due to the rapid growth of wireless communications and the development of miniaturized smart phones, the need for multisystem operation in a mobile device has become a necessity. Therefore, multiband antennas equipped in mobile devices are widely demanded. The frequency bands for mobile communication are GSM850 (824 894), GSM900 (890 960 MHz), DCS1800 (1710 1880 MHz), PCS1900 (1850 1990 MHz), and UMTS (1920 2170MHz). With the development of the $4 \mathrm{G}$ technology, there is an increasing requirement to additionally cover LTE2300 $(2305 \sim 2400 \mathrm{MHz})$ and LTE2500 (2500 2690MHz) bands.

Recently, many antenna configurations have been proposed to overcome the difficulties in the design of multiband and small size antennas. Some of them have tried to achieve a small size of structure. However, these antennas failed to cover the whole frequency bands required, especially at the lower frequency bands [1], [2]. Antennas with sufficient bandwidth require either a considerable antenna thickness or a considerable antenna height, making them unsuitable for mobile devices [3].

In this paper, we propose a novel monopole antenna with dimensions of $15 \mathrm{~mm} \times 40 \mathrm{~mm} \times 0.5 \mathrm{~mm}$. This antenna is capable of generating two wide operating bands to cover the GSM/UMTS/LTE operations in a mobile device. It is known that electromagnetic coupling and two-strip configurations are two effective methods for the bandwidth enhancement of a compact antenna structure [4], [5]. To achieve the wide bandwidth in the lower frequency, we design two electromagnetically-coupled strips that cover the lower band. An additional shorter meander branch is introduced for the 2.1

This work was supported by the NSFC (60871061), the GDSF (81510641010000085), the SRFDP (20080561), and the Oversea Distinguished Professor Program from the Ministry of Education of China.
$\mathrm{GHz}$ operation. The mutual coupling among the three strips helps in the bandwidth enhancement for the higher band without significant effect on the performance for the lower band. There is no shorting via involved in the antenna structure. The configuration and performance of the dual-frequency monopole antenna is described in section II. Experimental results are presented in section III.

\section{ANTENNA CONFIGURATION AND PERFORMANCE}

The configuration of the broadband antenna is illustrated in Fig. 1. The design of the antenna is based on a TLY-5 planar substrate that has a dielectric constant of $\varepsilon_{\mathrm{r}}=2.2$ and a thickness of $\mathrm{t}=0.5 \mathrm{~mm}$. The proposed antenna consists of a two-strip monopole for the $800 \mathrm{MHz}$ band and a meandered strip for the $2 \mathrm{GHz}$ band. The two-strip monopole is formed by an S strip and an inverted F strip. The meandered strip and the inverted strip are printed on the front side of the TLY substrate and fed by a $50 \Omega$ microstrip line. The $\mathrm{S}$ strip is etched on the backside of the substrate and terminated at a ground plane. The upper section of the inverted F strip is fitted into an area surrounded by the upper section of the $\mathrm{S}$ strip while the lower section of the inverted $\mathrm{F}$ strip overlaps with the lower section of the $\mathrm{S}$ strip, forming a two-strip line. The width (Wf) of the $50 \Omega$ feed line is $1.5 \mathrm{~mm}$ while the width (Wts) of the two-strip line is 1.2 $\mathrm{mm}$ to obtain a good impedance match. The meandered strip is connected to the feed line through a narrower microstrip line with its width (Wf2=1 mm) and length Lf2. The height $(\mathrm{H})$ of the two-strip line is the same as the total height of the antenna which equals $15 \mathrm{~mm}$.

The two-strip line is designed mainly to cover the lower band while its higher mode appears around $2.4 \mathrm{GHz}$. A meandered strip around $40 \mathrm{~mm}$ is added to generate a resonant mode around $1.9 \mathrm{GHz}$. As the configuration of the meander line is properly selected, good coupling among the three branches can enhance the bandwidth at the higher frequency without influence on the lower band.

To make the multi-band antenna have good impedance matching and sufficient bandwidth at the $900 \mathrm{MHz}$ and 1.9 $\mathrm{GHz}$ bands, the geometric parameters of the antenna need to be optimized.

The optimization design was carried out with the help of an extensive numerical simulation. The optimized values for the geometric parameters are listed in TABLE I. In the rest of the paper, all geometric parameters assume the values in this table 
unless they are given specifically. Fig. 2 shows the result of return loss for the optimal dual-band antenna. It is found that the bandwidths for voltage standing wave ratio (VSWR) $\leqslant 2.5$ at the $900 \mathrm{MHz}$ and $2 \mathrm{GHz}$ bands are $36 \%(0.8 \mathrm{GHz} 1.16 \mathrm{GHz})$ and $50 \%(1.70 \mathrm{GHz} 2.83 \mathrm{GHz})$, respectively.

It is noticed that the mutual coupling between the inverted F strip line and the S strip line has considerable influence on the bandwidth enhancement at the lower frequency. It enables the antenna to cover an extremely wide bandwidth around the $800 \mathrm{MHz}$ with a small size. The length of the inverted F strip is approximately $80 \mathrm{~mm}$ which is about $1 / 4$ wavelengths around $930 \mathrm{MHz}$ and the length of the coupling $\mathrm{S}$ strip line is approximately $120 \mathrm{~mm}$ which is about $1 / 4$ wavelength around $830 \mathrm{MHz}$. The combination of the two strips generates two resonant modes close each other. When the distance and the length of the two branches are properly selected, the two modes can be well mutually coupled and enhance the input impedance matching.

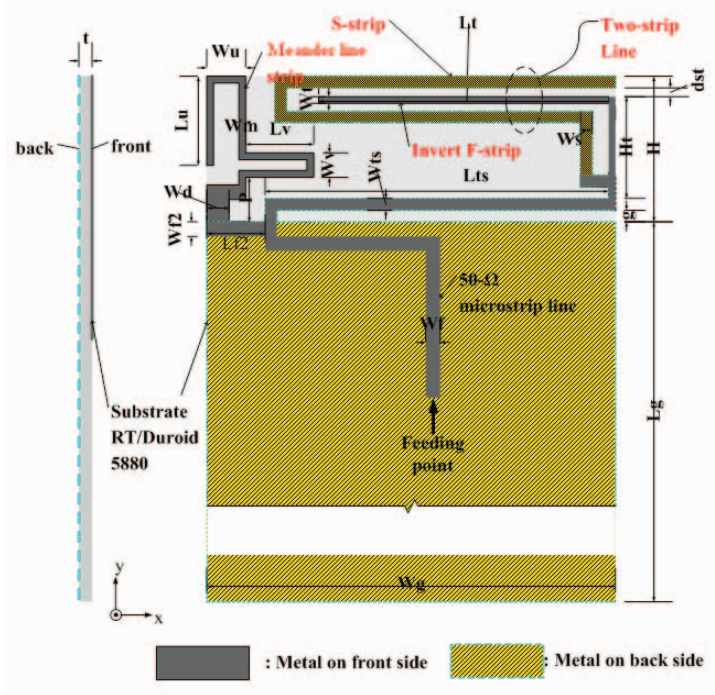

Fig. 1. Geometry of the dual-frequency broadband antenna. TABLE I Optimized values for the geometric parameters of the dualfrequency broadband antenna.

\begin{tabular}{|c|c|c|c|}
\hline parameter & value & parameter & value \\
\hline $\mathrm{H}$ & $15 \mathrm{~mm}$ & $\mathrm{Wu}$ & $4 \mathrm{~mm}$ \\
\hline $\mathrm{Lts}$ & $36 \mathrm{~mm}$ & $\mathrm{Lu}$ & $9.2 \mathrm{~mm}$ \\
\hline $\mathrm{Ht}$ & $10.5 \mathrm{~mm}$ & $\mathrm{Wv}$ & $2.5 \mathrm{~mm}$ \\
\hline $\mathrm{Lt}$ & $30 \mathrm{~mm}$ & $\mathrm{Wm}$ & $0.7 \mathrm{~mm}$ \\
\hline $\mathrm{Wt}$ & $0.7 \mathrm{~mm}$ & $\mathrm{Wd}$ & $2.3 \mathrm{~mm}$ \\
\hline $\mathrm{g}$ & $1.2 \mathrm{~mm}$ & $\mathrm{p}$ & $4.6 \mathrm{~mm}$ \\
\hline $\mathrm{t}$ & $0.5 \mathrm{~mm}$ & $\mathrm{Lv}$ & $7.5 \mathrm{~mm}$ \\
\hline $\mathrm{Ws}$ & $1.2 \mathrm{~mm}$ & $\mathrm{Wf}$ & $1.5 \mathrm{~mm}$ \\
\hline $\mathrm{Wf} 2$ & $1 \mathrm{~mm}$ & Wg & $42 \mathrm{~mm}$ \\
\hline $\mathrm{Lf} 2$ & $6 \mathrm{~mm}$ & Dst & $0.7 \mathrm{~mm}$ \\
\hline $\mathrm{Lg}$ & $100 \mathrm{~mm}$ & Wts & $1.2 \mathrm{~mm}$ \\
\hline
\end{tabular}

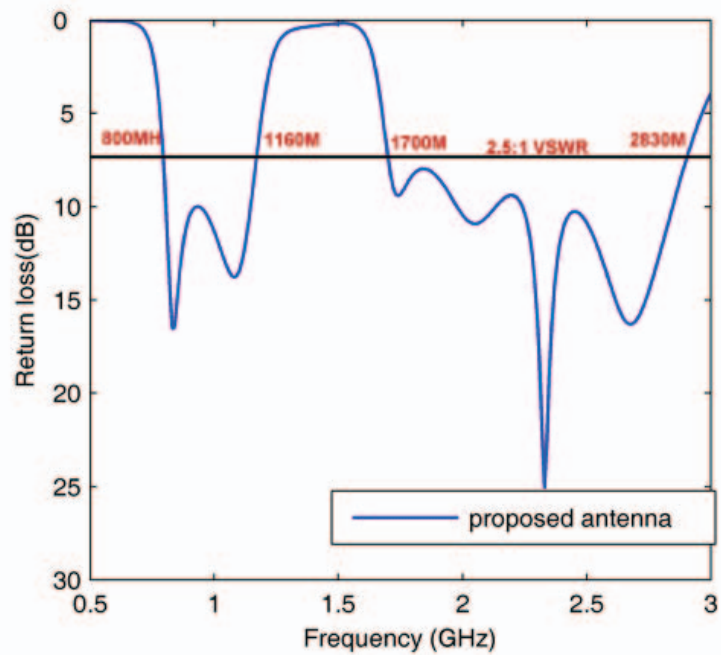

Fig. 2. Simulated return loss of the dual-frequency broadband antenna.

\section{IEXPERIMENTAL RESULTS}

To verify the performance of the compact broadband planar antenna, a prototype was fabricated and measured. The antenna was fabricated on a $0.5 \mathrm{~mm}$ TLY -5 substrate with $0.2 \mathrm{oz}$ copper on both sides. Two photographs of the antenna prototype are displayed in Fig. 3 which shows the front view and the back view of the planar antenna. For the purpose of measurement, the antenna is connected to a coaxial cable in the middle section of the ground plane. The measured return loss (RL) is presented in Fig. 4. It is clearly seen that two wide operating bandwidths are obtained. The lower bandwidth, determined by 1: 2 VSWR, reaches $372 \mathrm{MHz}(41 \%)$ and covers the GSM band $(824 \sim 960 \mathrm{MHz})$. On the other hand, the upper band, determined by 1: 2.5 VSWR, has a bandwidth as large as 1275 $\mathrm{MHz}(53 \%)$ and covers the GSM 1800 (1710 1880), GSM1900 (1850 1990), UMTS (1920 2170), LTE2300 (2305 2400), LTE2500 (2500 2690MHz) bands. The measured result agrees with the simulated result. The radiation patterns of the proposed antenna at the center frequencies for the lower and high bands are plotted in Fig. 5. At $900 \mathrm{MHz}$, a radiation pattern with omni-directional radiation in the azimuthal plane (x-z plane) is obtained while at $1900 \mathrm{MHz}$, variation in the patterns is observed due to high-order modes. The proposed antenna in general has a monopole-like radiation pattern. The measured peak gains at the frequencies of 900 , 1900 , and $2600 \mathrm{MHz}$ are approximately 1.4, 2.1, and $4.0 \mathrm{dBi}$, respectively.

\section{CONCLUSION}

The lower band of the proposed planar monopole has a large enough bandwidth to cover the frequency range from 818 $\mathrm{MHz}$ to $1190 \mathrm{MHz}$ for the GSM850/GSM900 operation. The upper band has an even larger bandwidth in order to cover the frequency range of $1710 \sim 3000 \mathrm{MHz}$ for the GSM1800 (1710 1880), GSM1900 (1850 1990), UMTS (1920 2170), LTE2300 (2305 2400), LTE2500 (2500 2690MHz) bands with the VSWR=2.5:1. The wideband dual-frequency antenna 
can be realized on a thin substrate without via process, thus facilitating its easy integration with RF front-end circuits for mobile devices.

\section{ACKNOWLEDGMENT}

The authors thank to SPEED WIRELESS TECHNOLOGY Co., Ltd for pattern measurement.

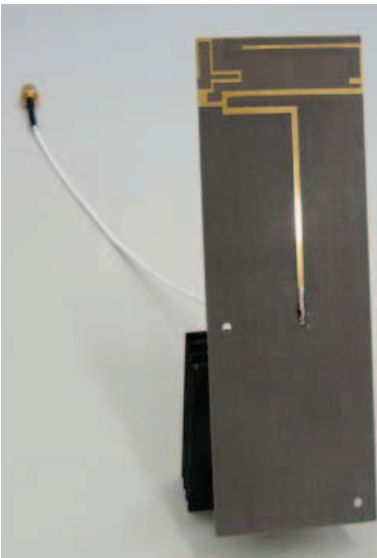

(a)

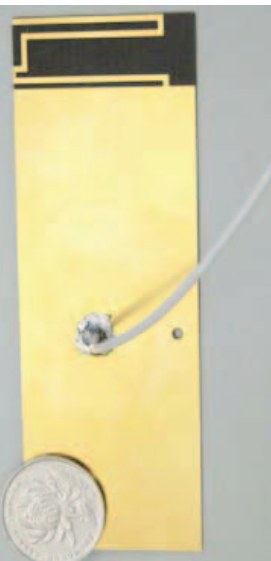

(b)
Fig. 3. Photographs of the dual-frequency broadband antenna with the optimized geometric parameters. (a) Front view. (b) Back view.

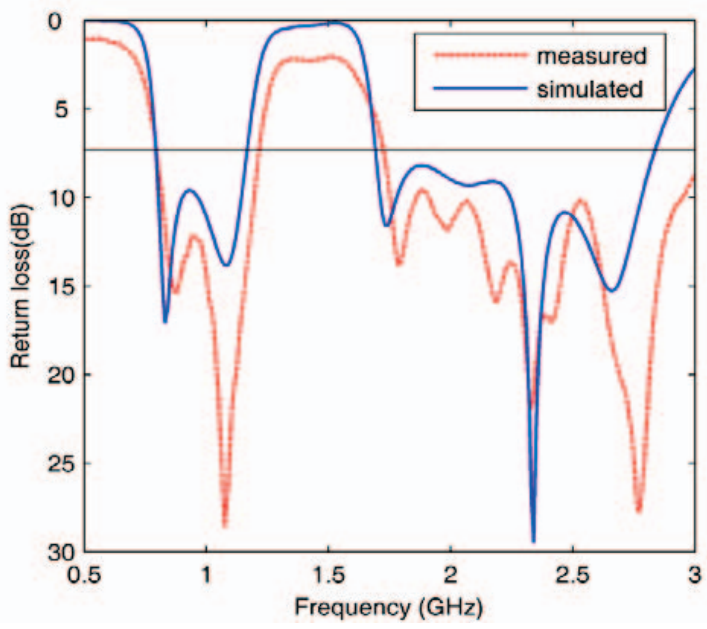

Fig. 4. Comparison of the measured and simulated result for return loss of the dual-frequency broadband antenna.
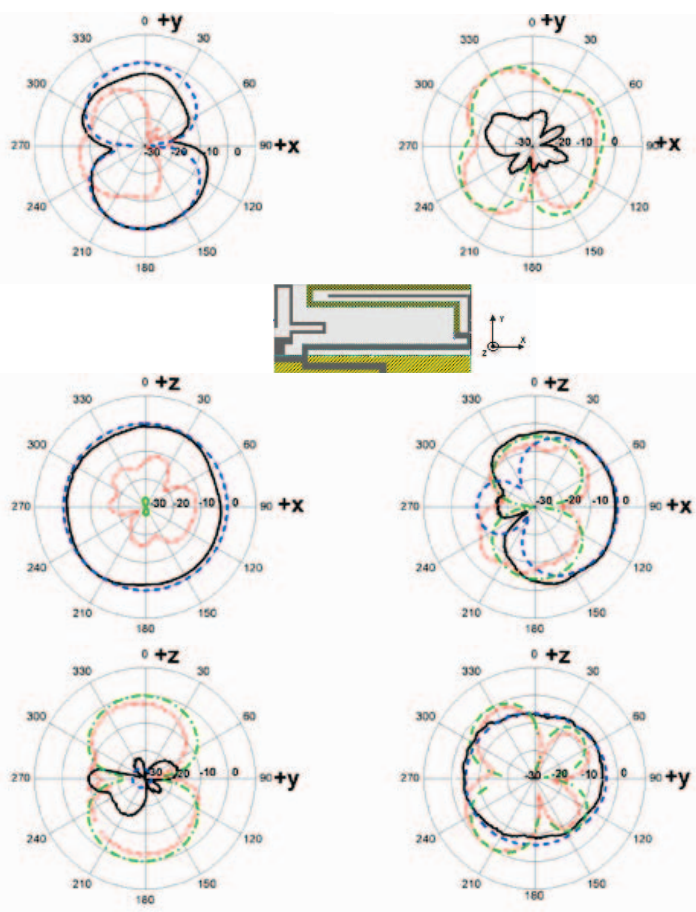

(b)

Fig. 5. Radiation patterns for the proposed antenna at: (a) $900 \mathrm{MHz}$, (b) $1900 \mathrm{MHz}$

\section{REFERENCES}

[1] Yun-Wen Chi, Kin-Lu Wong. "Quarter-Wavelength Printed Loop Antenna with an Internal Printed Matching Circuit for GSM/DCS/PCS/UMTS Operation in The Mobile Phone," IEEE Transactions on Antennas and Propagation, Vol. 57, No. 9, pp. 25412547, September. 2009.

[2] Seokjin Hong, Wonseob Kim, Hoon Park, "Design of an Internal Mult Resonant Monopole Antenna for GSM900/DCS1800/US-PCS/S-DMB Operation," IEEE Transactions on Antennas and Propagation, Vol. 56, No. 5, pp. 1437-1443, May 2008.

[3] Shun-Yun Lin, "Multiband Folded Planar Monopole Antenna for Mobile Handset," IEEE Transactions on Antennas and Propagation, Vol. 52, No. 7, pp. 1790-1794, July 2004.

[4] RongLin Li, Bo Pan, Joy Laskar, and Manos M. Tentzeris, "A Compact Broadband Planar Antenna for GPS, DCS-1800, IMT-2000, and WLAN Applications," IEEE Antennas and Wireless Propagation Letters, Vol. 6, pp. 25-27, 2007.

[5] RongLin Li, Bo Pan, Joy Laskar, and Manos M. Tentzeris, "A Novel Low-Profile Broadband Dual-Frequency Planar Antenna for Wireless Handsets," IEEE Transactions on Antennas and Propagation, Vol. 56, No. 4,pp. 1155-1162, April 2008 